University of Louisville

ThinkIR: The University of Louisville's Institutional Repository

\title{
Bipolar cell dendritic morphology analysis in mice with model retinitis pigmentosa.
}

Simra Ahmed

Follow this and additional works at: https://ir.library.louisville.edu/honors

Part of the Biology Commons

\section{Recommended Citation}

Ahmed, Simra, "Bipolar cell dendritic morphology analysis in mice with model retinitis pigmentosa." (2016). College of Arts \& Sciences Senior Honors Theses. Paper 115.

http://doi.org/10.18297/honors/115

This Senior Honors Thesis is brought to you for free and open access by the College of Arts \& Sciences at ThinkIR: The University of Louisville's Institutional Repository. It has been accepted for inclusion in College of Arts \& Sciences Senior Honors Theses by an authorized administrator of ThinkIR: The University of Louisville's Institutional Repository. This title appears here courtesy of the author, who has retained all other copyrights. For more information, please contact thinkir@louisville.edu. 


\section{Bipolar Cell Dendritic Morphology Analysis}

in Mice with Model Retinitis Pigmentosa

\section{By}

\section{Simra Ahmed}

Submitted in partial fulfillment of the requirements for Graduation summa cum laude

and

for Graduation with Honors from the Department of Biology University of Louisville

May, 2016 


\section{Background}

The eye is a complex structure with many working parts that each has a complexity of their own. The retina is arguably one of the most complex parts of the eye, anatomically and functionally, being only a single layer of tissue. It is posteriorly located in the eye, and is the innermost covering of the back of the eye, directly in contact with the vitreous chamber and vitreous fluid. Light and images are focused through the lens of the eye and are then projected on the retina, which are then processed through the retina and sent via electrical signals to the optic nerve then brain for mental processing. Infamously known for its composition of rods and cones, the retina is actually composed of many separate layers with multiple different cell types that are part of an intricate chain of signal communication. For a general understanding of the layout of the retina, beginning with the innermost layer of the retina, there is the ganglionic cell layer, the inner plexiform layer, the inner nuclear layer, the outer plexiform layer, the outer nuclear layer, and the pigment epithelium. [1]. As can be seen in figure 1,

\begin{tabular}{|l|l|}
\hline Layer & Contains: \\
\hline Outer nuclear layer (ONL) & $\begin{array}{l}\text { Photoreceptors, rods, and } \\
\text { cones }\end{array}$ \\
\hline Inner nuclear layer (INL) & $\begin{array}{l}\text { Horizontal cells, bipolar cells, } \\
\text { amacrine cells, Müller cells }\end{array}$ \\
\hline Ganglion cell layer (GCL) & $\begin{array}{l}\text { Ganglion cells, displaced } \\
\text { amacrine cells }\end{array}$ \\
\hline Outer plexiform layer (OPL) & $\begin{array}{l}\text { Photoreceptors talk to } \\
\text { horizontal cells and bipolar cells }\end{array}$ \\
\hline Inner plexiform layer (IPL) & $\begin{array}{l}\text { Bipolar cells talk to amacrine } \\
\text { cells and ganglion cells }\end{array}$ \\
\hline
\end{tabular}

the various retinal

cells are

strategically

organized in layers

within the retina.

For this study in

Figure 1: A table of the various layers in the retina and the specific retinal cells within those layers [1].

particular, the focus is bipolar cells and 
cone photoreceptors; for referential understanding, figure 2 shows the bipolar cell

dendrites contacting

cone terminals in the

outer plexiform layer

(OPL).

Bipolar cells are

a perfect

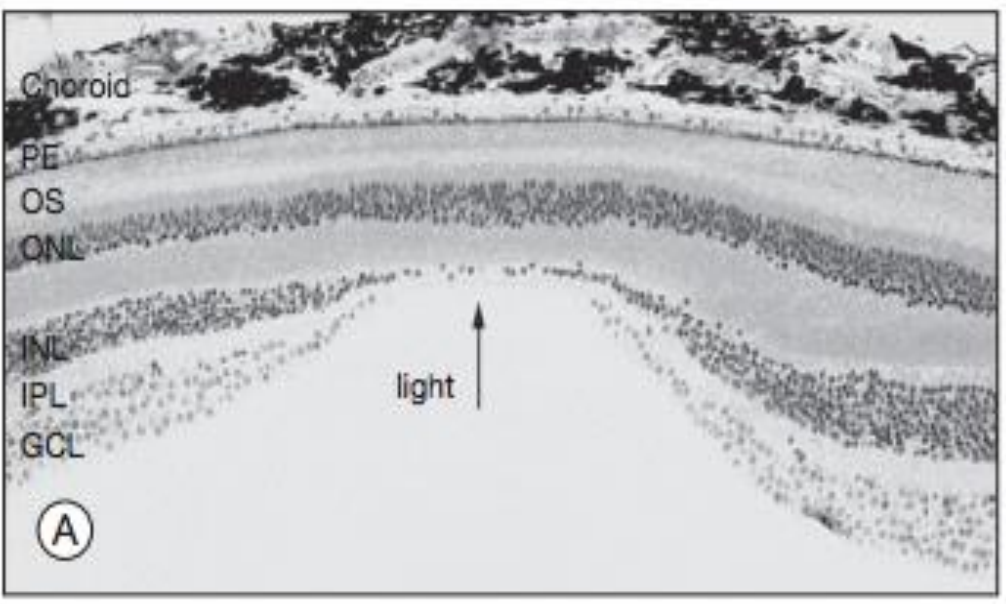

demonstration of the

complexity of the

retina; up to date, 10 -

12 different types of

bipolar cells have been

discovered and

extensively researched.

There is one type of rod

bipolar cell (which is an

ON bipolar cell) while all

other bipolar cells are

considered cone bipolar

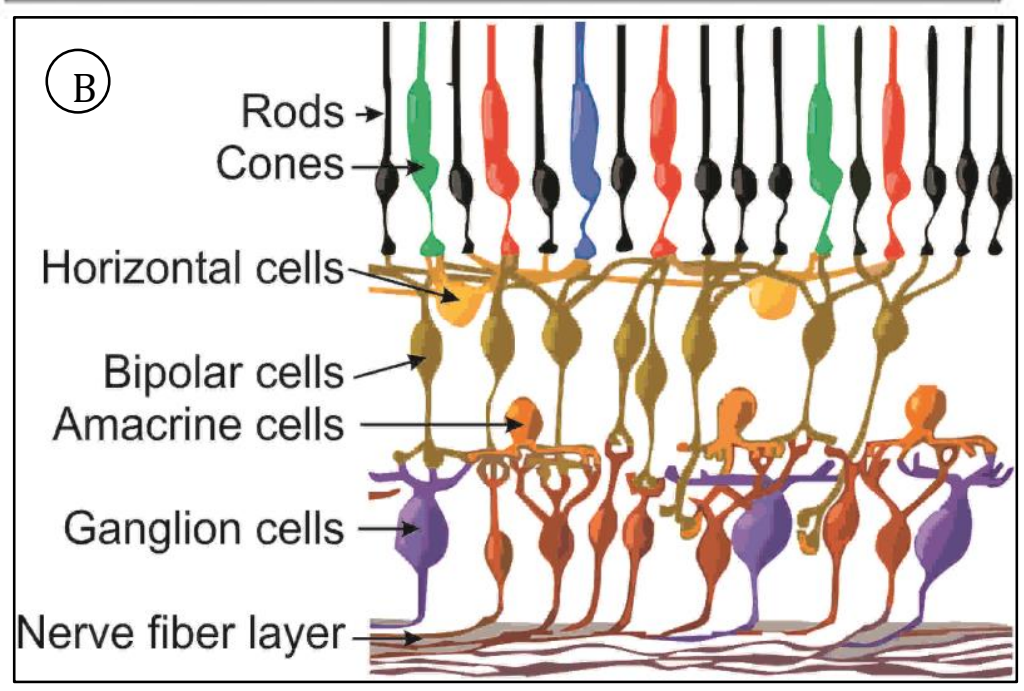

Figure 2: (A) A lateral slice of the retina, which shows clear delineations of the different layers. The outer plexiform layer (OPL) is unlabeled, but is between the layers labeled inner nuclear layer (INL) and outer nuclear layer (ONL) [1]. (B) Illustrates the bipolar cell dendrites contacting the photoreceptors in between the outer plexiform layer (OPL) and the outer nuclear layer (ONL), containing the photoreceptors.

cells. Rod and cone bipolar cells are named according to their presynaptic partners the rod and cone photoreceptors. The cone bipolar cells can be further subdivided into ON and OFF bipolar cells. ON bipolar cells are depolarized by light. Oppositely, OFF bipolar cells are hyperpolarized by light. The other main 
distinction between ON and OFF bipolar cells is their stratification in the inner plexiform layer (IPL); the OFF bipolar cells do not penetrate the IPL as deep as the ON bipolar cells and their axonal branches usually terminate in sublamina a. While ON bipolar cells axonal branches usually stratify further down in the IPL, into sublamina $b$ [1], shown in figure 3. Additionally, each ON and OFF cone bipolar cell contacts ganglion cells and provide their excitatory input, hence the ON bipolar cells contact ON retinal ganglion cells, whilst the OFF bipolar cells contact the OFF bipolar cells. Multiple lines of transgenic mice have been developed that express a fluorescent tracer in specific types of bipolar cells[2]. In this study, VSX mice and GusGFP mice were tested; the VSX mice labels for Off Type 1 and 2 bipolar cells [3] while the GusGFP mice label On Type 7 and rod bipolar cells [2].

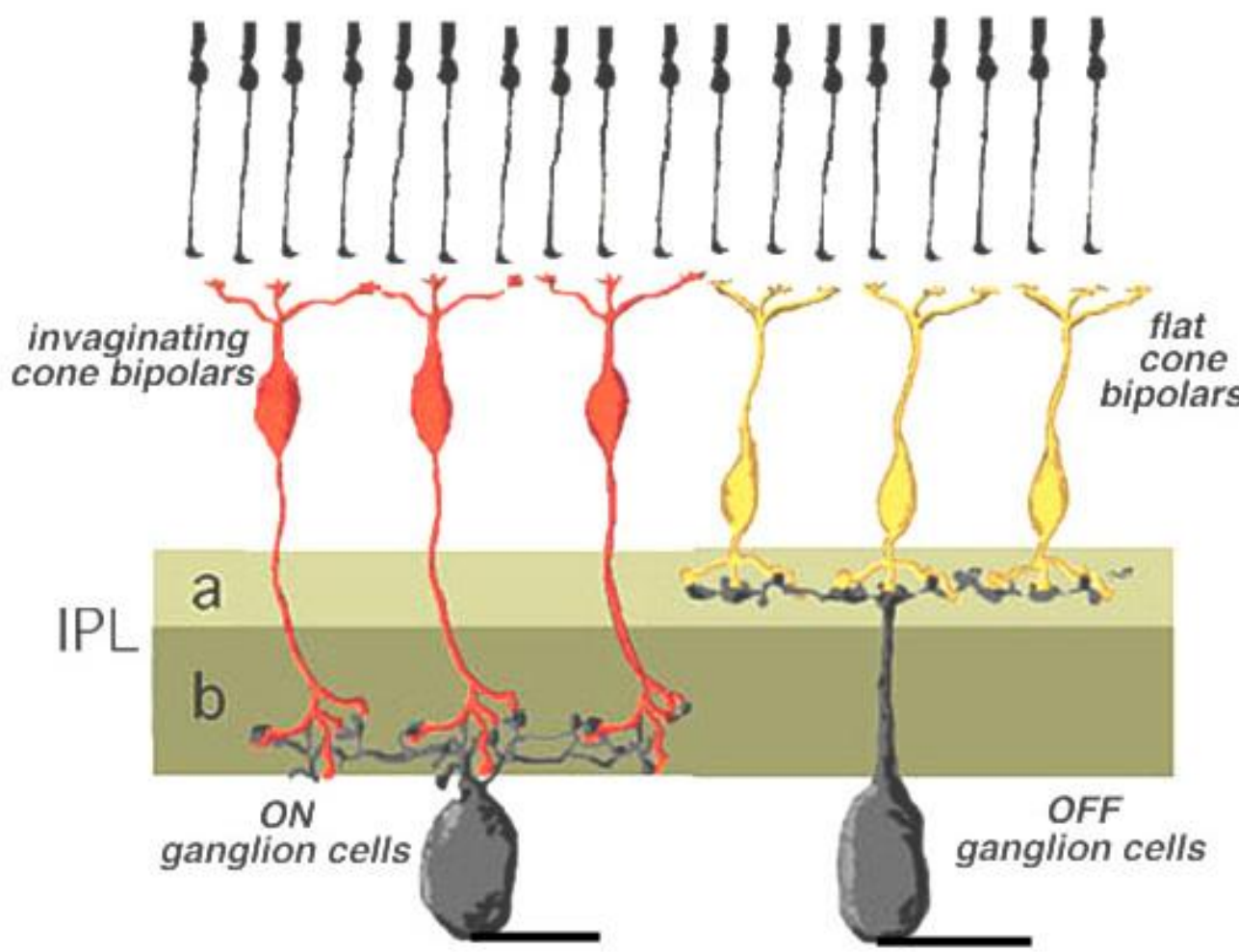

Figure 3: This schematic drawing of cone bipolar cells shows their dendrites contacting the cones in the division between the top layers. Additionally, the drawing shows the ON bipolar cells (in orange) stratifying into sublamina $b$ of the IPL where they are contacting $\mathrm{ON}$ ganglion cells, and the OFF bipolar cells, in yellow, are shown contacting the OFF ganglion cells higher up in the IPL at sublamina $A$ [11]. 
Retinitis

Pigmentosa (RP) is a fairly widespread retinal disease that results from a range of mutations in proteins expressed in rod photoreceptors. It has an estimated incident rate in the United States of 1:3,500
A

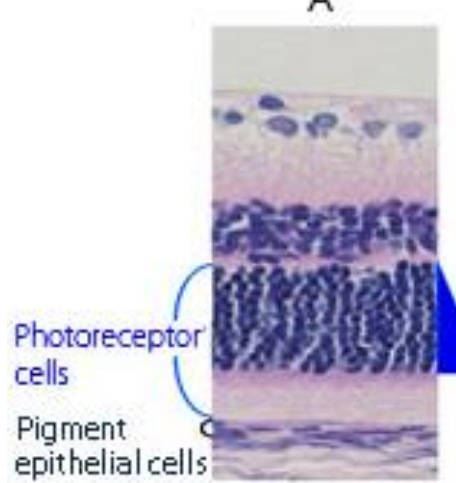

Untreated week 3
B

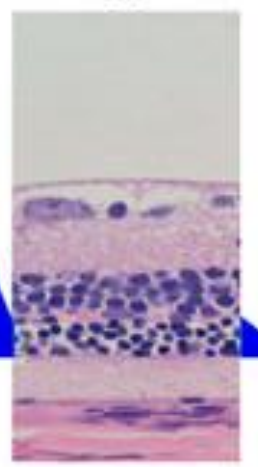

Untreated week 5
C

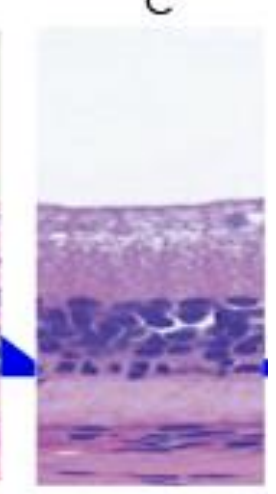

Untreated I week 7

Figure 3: These three images illustrate the progressive deterioration of the photoreceptors in rat retinas over time [12].

births[3]. RP is a degenerative disease in which the rod photoreceptors die first, which leads to an initial defect in night vision. Later for reasons that still are not clear, cone photoreceptors also degenerate, as pictured in figure 4. This leads to progressive deterioration of vision and finally to blindness. In advanced stages of retinitis pigmentosa, there is evidence of limited damage to the ganglion, bipolar, and amacrine cells[4]. The leading cause of RP in North America is a result of an autosomal dominant mutation in the rhodopsin gene in which there is a proline to histidine substitution at codon $23(\mathrm{P} 23 \mathrm{H})[3]$. Rhodopsin is the visual pigment in rod photoreceptors that aids vision in $\operatorname{dim}$ lighting, and is found as a vitamin $A$ derivative11-cis retinal. After activation, the rhodopsin becomes all-trans retinal, and failure of cellular mechanisms to convert the all-trans back to 11-cis in the retinoid cycle are causes of retinal diseases and degeneration, like retinitis pigmentosa [5]. The $\mathrm{P} 23 \mathrm{H}$ knock in mouse (P23H KI), in which one copy of the 
rhodopsin gene is replaced by the $\mathrm{P} 23 \mathrm{H}$ mutation, has been a useful tool for investigating RP[6]. In both mice and humans, the $\mathrm{P} 23 \mathrm{H}$ mutation has shown comparable models of retinal degeneration. Also, mice are a suitable model for research studies because of their shorter generation times and easy genetic manipulation.[7]

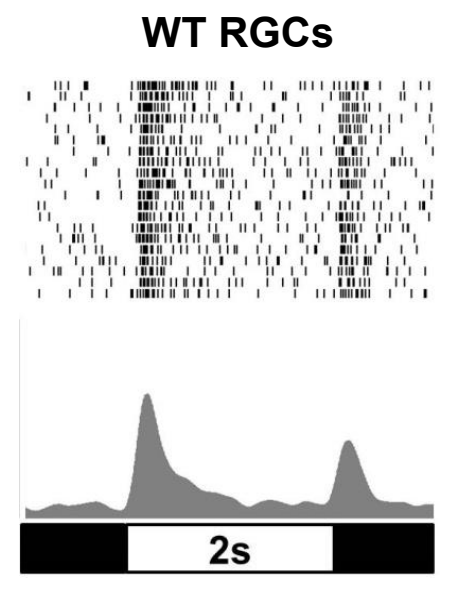

P23HKI RGCs

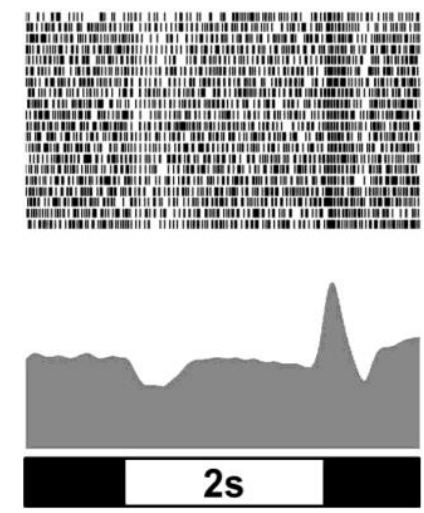

Figure 4: The suppressive response shown in the mutated P150 mice can be seen in the ERG results, as compared to the initial excitatory response seen in the WT.
Evidence in preliminary data indicates that there are changes in the visual output of the $\mathrm{P} 23 \mathrm{H} \mathrm{KI}$ mouse. Gobinda Pangeni, a postdoctoral fellow in the McCall lab has examined retinal function in the $\mathrm{P} 23 \mathrm{H}$ $\mathrm{KI}$ mouse between postnatal day (P) 30, P90, and P150 by recording the visually evoked responses of the retinal ganglion cells (RGC), the output cells of the retina. He has noted changes in $\mathrm{RGC}$ visual function that suggest that the input from the cone pathways of some ganglion cells are more vulnerable to the effects of rod and cone photoreceptor degeneration and lose excitatory inputs. The RGC excitatory inputs come from bipolar cells, and the inputs are segregated so that bipolar cells that are excited to a luminance increase provide excitatory input to RGCs with similar responses. In contrast, bipolar cells that are excited to a luminance decrease provide excitatory input to RGCs that are also excited by the same stimulus. The 
lab has noted that over a period of 150 days some P23H mutant RGCs show a suppression of to onset of light rather than an excitation, which is found in WT RGCs. This most likely indicates the elimination of an excitatory input, which is present in the normal RGC. This also could reflect a reorganization of inputs to the RGC, but these changes would occur in the direct circuits that are provided by inhibitory interneurons of the retina, the amacrine cells. A change in excitatory input could reflect: (1) a decrease in the number of bipolar cell contacts with the cone photoreceptors, or (2) the loss of dendrites or shrinkage of the dendritic area in bipolar cells.

This data has led to the question whether there is a deficiency or degeneration in the ratio of bipolar cell dendrites to cone contact terminal contact. My hypothesis was that the changes in visual function could at least be explained in part with changes in the morphology of the bipolar cell dendrites. The hypothesis was tested by examining the number of bipolar cell contacts with cone photoreceptor terminals at different ages: postnatal days 90 and 150 . Additionally, the $\mathrm{P} 23 \mathrm{H} \mathrm{KI}$ retina was compared with control retinas (wild type mice) to determine if any of the bipolar cell classes show change in the mutated model's dendritic length, branch points, and or dendritic area.

My Specific Aim was to:

\section{Determine if there are changes in the morphology of bipolar cells in mouse}

\section{retinas with retinitis pigmentosa.}

In order to determine morphological changes in the bipolar cells, I measured dendritic area, dendritic length, and number of dendritic contacts with cone 
photoreceptors in the labeled bipolar cells and analyzed. Cell data was collected based on the assumption that if there is significant change in the mutated model of bipolar cells it occurs homogeneously throughout the spatial organization of the retina. Because the mouse retina has no specialization and is a representation of the human peripheral retina, no pattern of degeneration was anticipated and cells for measurement were randomly chosen from the retinas. Additionally, a study done shows photoreceptors follow a pattern of degeneration from the center of a retina towards the periphery, but cone bipolar cells degenerate independently of this pattern. The study first examined ON Type 7 cone bipolar cells in the mouse model of retinitis pigmentosa and later expanded the research to all types of cone bipolar cells. From the data it was concluded that the cone bipolar cells do not follow the spatiotemporal degeneration pattern of cones (center to peripheral degeneration) and that there is uniform spontaneous degeneration of bipolar cells in the mice with retinitis pigmentosa [13]. The $\mathrm{P} 23 \mathrm{H} \mathrm{KI}$ data of each tested mouse line was compared with its own specific control littermate's data. The results determined whether changes in bipolar cell dendritic morphology do exist and in turn might account for the observed change in visual output that has been previously seen.

\begin{tabular}{|l|l|}
\hline Reporter Line/ Transgene & Bipolar Cell labeled \\
\hline VSX1 & Off BC - Type 1 and 2 \\
\hline Gus GFP & On BC - Type 7 and rod bipolar cells \\
\hline
\end{tabular}

Table 1: The table above shows the various bipolar cells labeled in the particular mice reporter lines used for this study. 


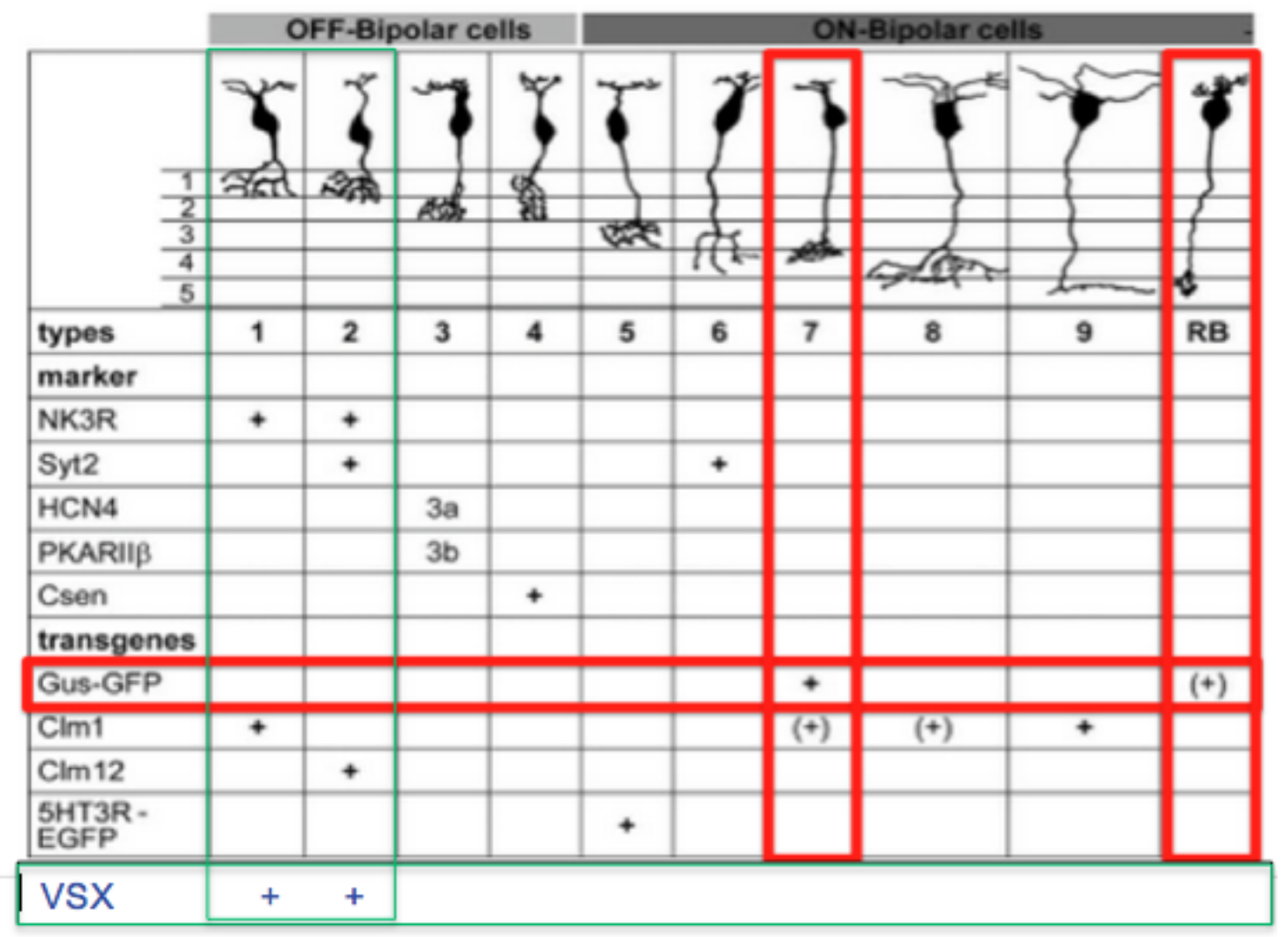

Figure 6: The table below shows the results of a study that was conducted in order to determine which reporter lines label which bipolar cells, and a visual representation of the different types of bipolar cells can be seen above the table. [2] This table shows that the Gus-GFP mice stain for ON cone Type 7 and ON rod bipolar cells, and the VSX mice stain for OFF Type 1 and Type 2 bipolar cells.

\section{Significance}

The mouse model of retinitis pigmentosa in the $\mathrm{P} 23 \mathrm{H} \mathrm{KI}$ mice is very similar to that of the human form of retinitis pigmentosa: any discoveries made about this disease in the mouse model can provide insight into the happenings of RP patients that share the same mutation. The mouse model is an excellent representation of the peripheral retina in humans, but not the fovea since mice lack a fovea. The incident rate for this disease is relatively high; in turn there is great value in research behind the cause or potential enhancers of the disease. 
In humans, the disease onset occurs in the late teens early twenties and progresses throughout life. Because in retinitis pigmentosa there is an increasingly continual loss of rod and cone photoreceptors throughout life, a typical chronological progression of the disease would begin with loss of night vision early on in life, next loss of peripheral vision, and eventually loss of central vision[8]. Symptoms most often include night blindness and decreased visual acuity. Discovering explanations as to what could be a potential cause for RP is the first vital step in being able to discover treatments and/or cures for the disease.

\section{Experimental Approach - Methodology}

Animals. Mice were bred in accordance to University of Louisville's Institutional Animal Care and Use Committee's regulations. P23H KI mice [9] were bred to specific transgenic mice reporter lines: GusGFP and VSX (See Figure 6). Additionally, wild type transgenic mice, (those that do not carry the $\mathrm{P} 23 \mathrm{H}$ mutation) mice were used as controls. Mice of either sex aged $\sim 90$ days and $\sim 150$ days were used. After induction of anesthesia, a $60 \%$ ketamine and $40 \%$ xylazine anesthetic was used, a light was shone in each eye of the mouse for one minute. This elicits the movement of cone arrestin to the end of the cone terminals, which enhances their visualization. The mice were euthanized by anesthetic overdose, and death was assured by cervical dislocation. The eyes were enucleated and prepared for retinal dissection. 
Retinal Preparation and Immunohistochemistry. Eyes were placed in Ringer's solution, the front of the eye and the lens were removed and the retina removed from the posterior eyecup. After retinal dissection, the retina was placed on filter paper photoreceptor side down and dried for 15 seconds in order to adhere the retina to the filter paper. The retinas were fixed for 20-45 minutes in $4 \%$ paraformaldehyde in phosphate buffer saline. After fixation was completed, the retina was washed for ten minutes three times in $0.1 \mathrm{M}$ PBS. The retina was washed in $0.01 \mathrm{M}$ PBS and $0.5 \%$ Triton X-100 (PBX) for 45 minutes and reacted in blocking solution (a 0.01 M PBS, $0.5 \%$ Triton X-100 and 5\% Normal Goat Serum (NGS)) for one hour. Table 3 shows the various reagents used in these immunohistochemistry experiments, their concentrations and manufacturers. The retina was then reacted with primary antibodies chicken Anti-GFP (1:1000; Molecular Probes, Eugene, OR: A11120) and rabbit Anti-Mouse Cone Arrestin (1:1000; gift from Mary Allen Laboratory, Los Angeles, CA) diluted in blocking solution that label the desired proteins for fluorescence for $7-10$ days at $4^{\circ} \mathrm{C}$. The VSX1 reporter mouse expresses the CFP in its OFF Type 1 and Type 2 bipolar cells[10], whereas the GusGFP mouse expresses GFP in its ON Type 7 and rod bipolar cells[2]. The chicken Anti-GFP antibody, which is not conjugated, recognizes the fluorescence protein that fills the respective bipolar cells' cytoplasm and is used to image the bipolar cells on the confocal microscope. The rabbit Anti- Mouse Cone Arrestin primary antibody, which is not conjugated, recognizes the cone arrestin protein in the cone pedicels of both lines of mice. 


\begin{tabular}{|l|l|l|l|l|l|}
\hline $\begin{array}{l}\text { Mouse } \\
\text { Transgenic } \\
\text { Line }\end{array}$ & Age & $\begin{array}{l}\text { WT or } \\
\text { Mutant }\end{array}$ & $\begin{array}{l}\text { Number } \\
\text { of Mice }\end{array}$ & $\begin{array}{l}\text { Number of } \\
\text { Retinas } \\
\text { Used }\end{array}$ & $\begin{array}{l}\text { Number of } \\
\text { Cells }\end{array}$ \\
\hline VSX1 & P90 & WT & 2 & 4 & 11 \\
\hline VSX1 & P90 & P23H & 3 & 2 & 9 \\
\hline VSX1 & P150 & WT & 1 & 2 & 10 \\
\hline VSX1 & P150 & P23H & 1 & 2 & 13 \\
\hline GusGFP & P150 & WT & 2 & 2 & 7 \\
\hline GusGFP & P150 & P23H & 3 & 4 & 14 \\
\hline
\end{tabular}

Retinas were then washed six times $0.1 \mathrm{M}$ PBS for ten minutes each Table 2: Shows the number of mice used for the experiments and the number of corresponding retinas that were usable after dissection and immunohistochemistry and the amount of cells that came from those retinas.

wash, and then incubated in $200 \mathrm{uL}$ of secondary antibody solution overnight in the dark at $4^{\circ} \mathrm{C}$. The secondary antibody solution consisted of $0.1 \mathrm{M}$ PBS and secondary antibodies [goat anti-chicken, Alexa Fluor 488 (1:1000; Life Technologies, Eugene, OR) and goat anti-rabbit, Cy3-conjugated AffiniPure Alexa Fluor 546 (1:1000; Jackson Immuno Research, West Grove, PA: 111-165003)]. The goat anti-chicken secondary antibody recognizes the labeled green fluorescence protein and tags it with the 488 fluorescence, while the goat antirabbit secondary antibody recognizes the labeled cone arrestin and fluoresces it at the 546 wavelength. After leaving the retinas overnight, minimum of 12 hours no more than 24 hours, they were, once again, washed six times in 0.1 M PBS for ten minutes each wash. The whole mount retinas were mounted with Vectashield (Vector Laboratories, Burlingame, CA), and the slides were labeled 
WT or $\mathrm{P} 23 \mathrm{H}$, cover slipped, and sealed with nail lacquer. For optimal imaging area, extra precaution was exercised so the retina remained as flat as possible with minimal folds and retinas were kept on filter paper when mounted.

\begin{tabular}{|l|l|l|l|l|}
\hline Antibody & & Ratio & Source & Catalog Number \\
\hline Chicken Anti-GFP & Primary & $1: 1000$ & $\begin{array}{l}\text { Molecular Probes,; } \\
\text { Eugene, OR }\end{array}$ & A11120 \\
Rabbit Anti-Moue & Primary & $1: 1000$ & Mary Allen & \\
Cone Arrestin & & & Laboratory; Los & \\
Goat Anti-Chicken & Secondary & $1: 1000$ & Angeles, CA & \\
Alexa Fluor 488 & & & Life Technologies; & \\
\hline Cy3 - conjugated & Secondary & $1: 1000$ & Eugene, OR & \\
AffiniPure Goat & & & Jackson Immuno & $111-165-003$ \\
Anti-Rabbit Alexa & & & Research,;West & \\
Fluor 546 & & & Grove, PA & \\
\hline
\end{tabular}

Table 3: Shows the antibodies used in this study and their respective details. Confocal Microscopy and Imaging of Bipolar Cells. Retinal bipolar cells images were obtained using an Olympus laser scanning confocal microscope (Olympus FV1000) and imaging software (Fluoview FV1000). Bipolar cells were imaged from cell body to dendritic branches as were the cone photoreceptors. All images were taken using 1.35 NA 60x - oil magnification objective. All settings used when taking images for this study were as follows: aspect ratio 1:1, zoom 1.8-3.0, 4.0 usec/pixel, 1024x1024, and 0.4 um step size. Representative images of the VSX and GusGFP bipolar cells are shown in Figure 7. Table 2 shows the number of mice used and the number of cells from each particular type of mouse that were used. 
Image Analysis. I measured dendritic length and dendritic branch points, from the confocal images of isolated bipolar cells using Imaris 8.1 software. I used the automatic filament tracer option and made manual edits, like deleting false dendritic spines or adding in missed dendrites to adjust program errors. Then the individual dendrites of the bipolar cells were skeletonized as shown in Figure 8.

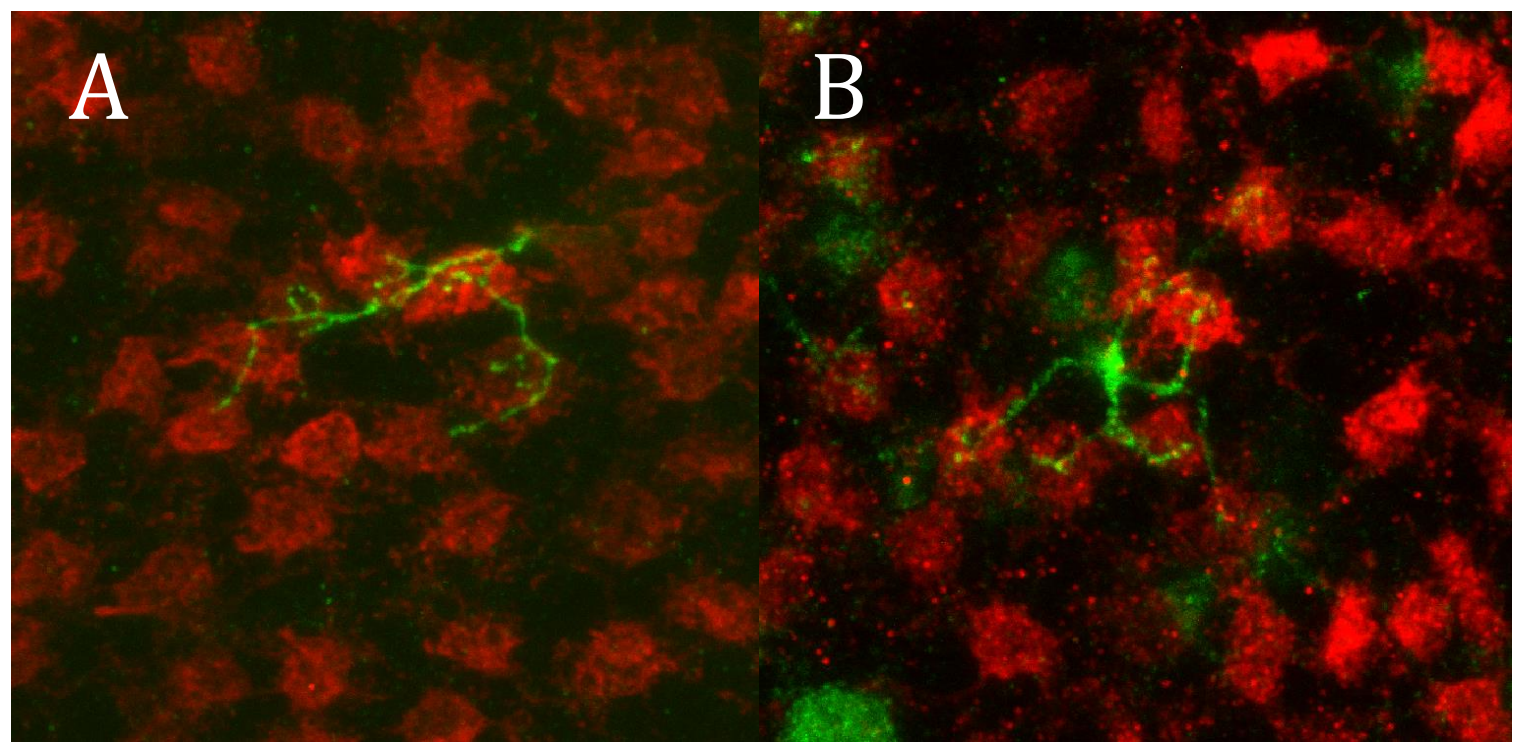

Figure 7 A) Shows an image of a VSX bipolar cell's dendrites. (B) Shows an image of a GusGFP bipolar cell's dendrites.

To measure dendritic area the two-dimensional bottom-view picture of the individual skeletonized dendrites of a bipolar cell generated in the Imaris Software was run through ImageJ software and the outer perimeter of the cell was manually traced excluding the primary dendrite and automatically measure, shown in Figure 9. The confocal Z-stack images, which are a cumulative stack of all the transverse sections of a retina overlapped into a single two-dimensional image, were processed using Adobe Photoshop CS4 and fluorescence of the bipolar cell dendrites and cone terminals were enhanced to determine areas of 
overlap.

Figure 5: Shows the skeletonized dendrites of a P23H KI Het ${ }^{+/}$VSX P90 mouse generated through Imaris. (A) Shows the bottom-view of the dendrites, while image (B) shows the side view of the 3-dimensional skeletonized bipolar cell's dendrites.

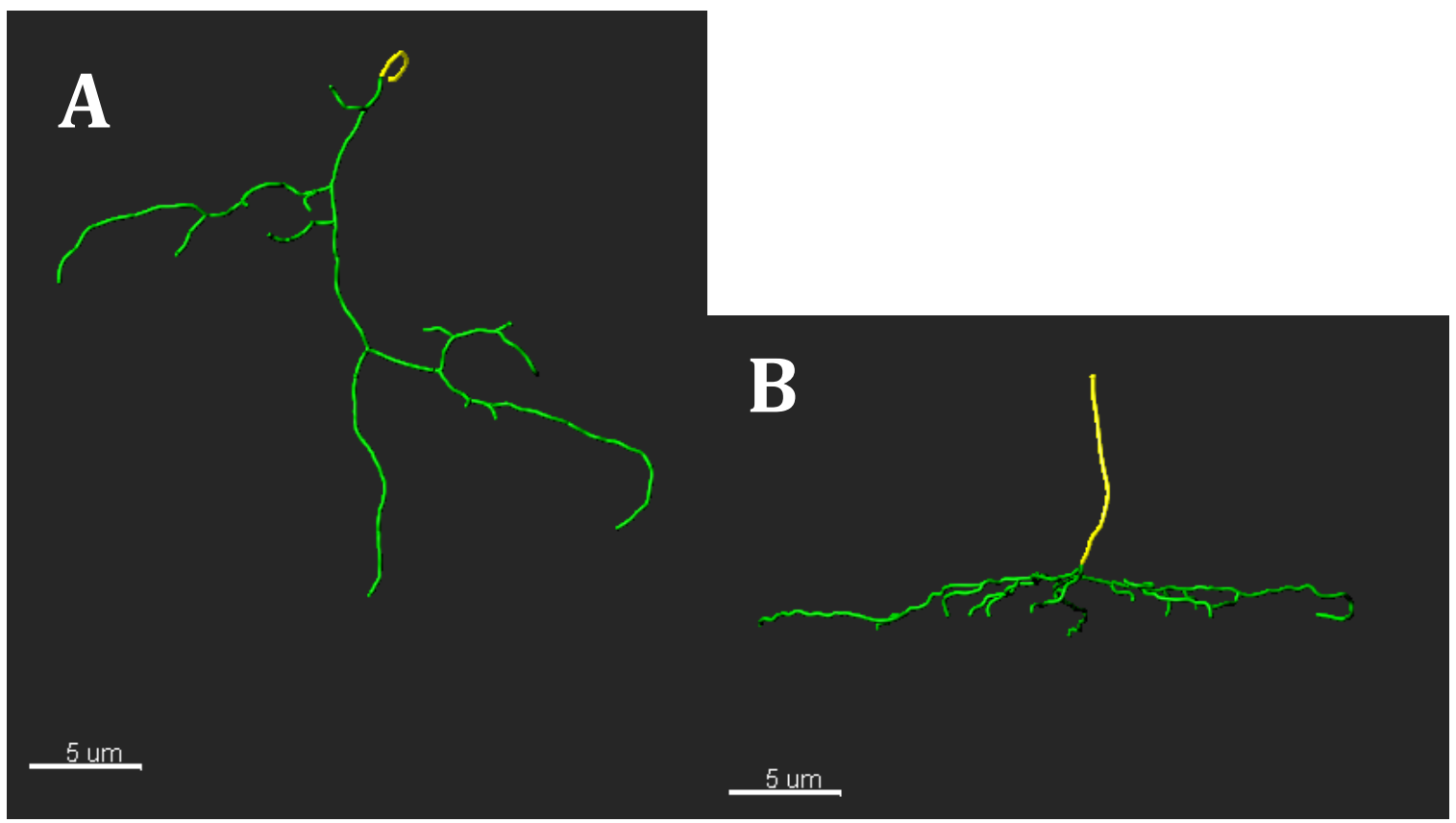

The contacts between bipolar cell dendrites and cone terminals were

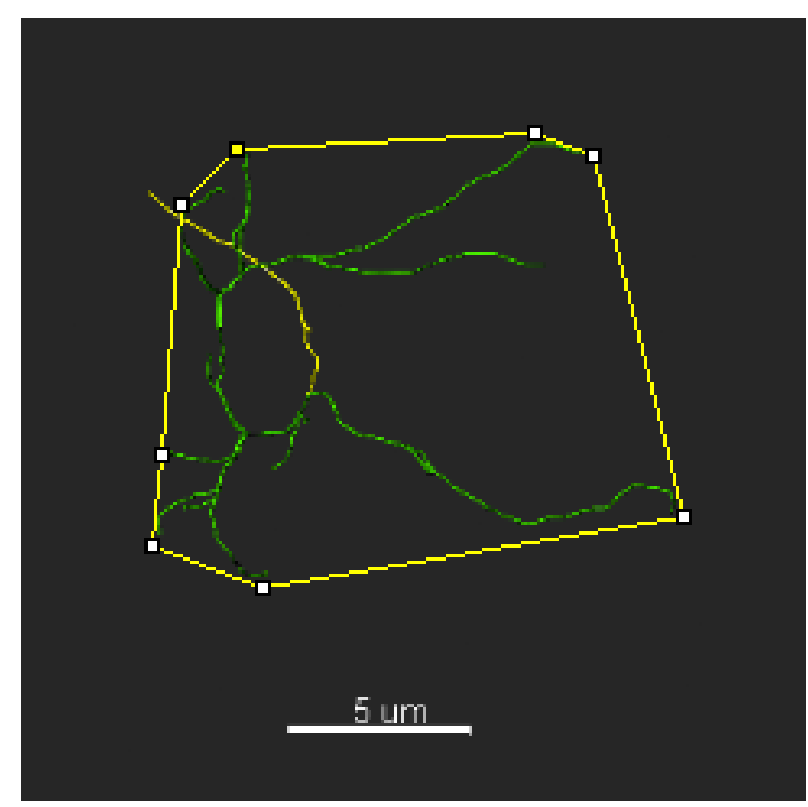

quantified from the confocal z-stack

images with the primary dendrite and

cell body masked (Figure 10). The

cone contacts were double-checked by

overlapping the Imaris skeletonized

dendrites onto the confocal z-stack to

ensure the dendritic spread was

accurately estimated. 
The skeletonized Imaris generated bipolar cell side view was compared to the z-

stack to verify that the dendritic branches reached into the cone layer and

Figure 6: This is an image showing the bottom-view of the skeletonized dendrites actually contact the cone terminals because twobeing run through ImageJ for area analysis. The area of this dendrite's cell dimensional top down views and bottom down views was $\sim 136 \mathrm{um}^{2}$.

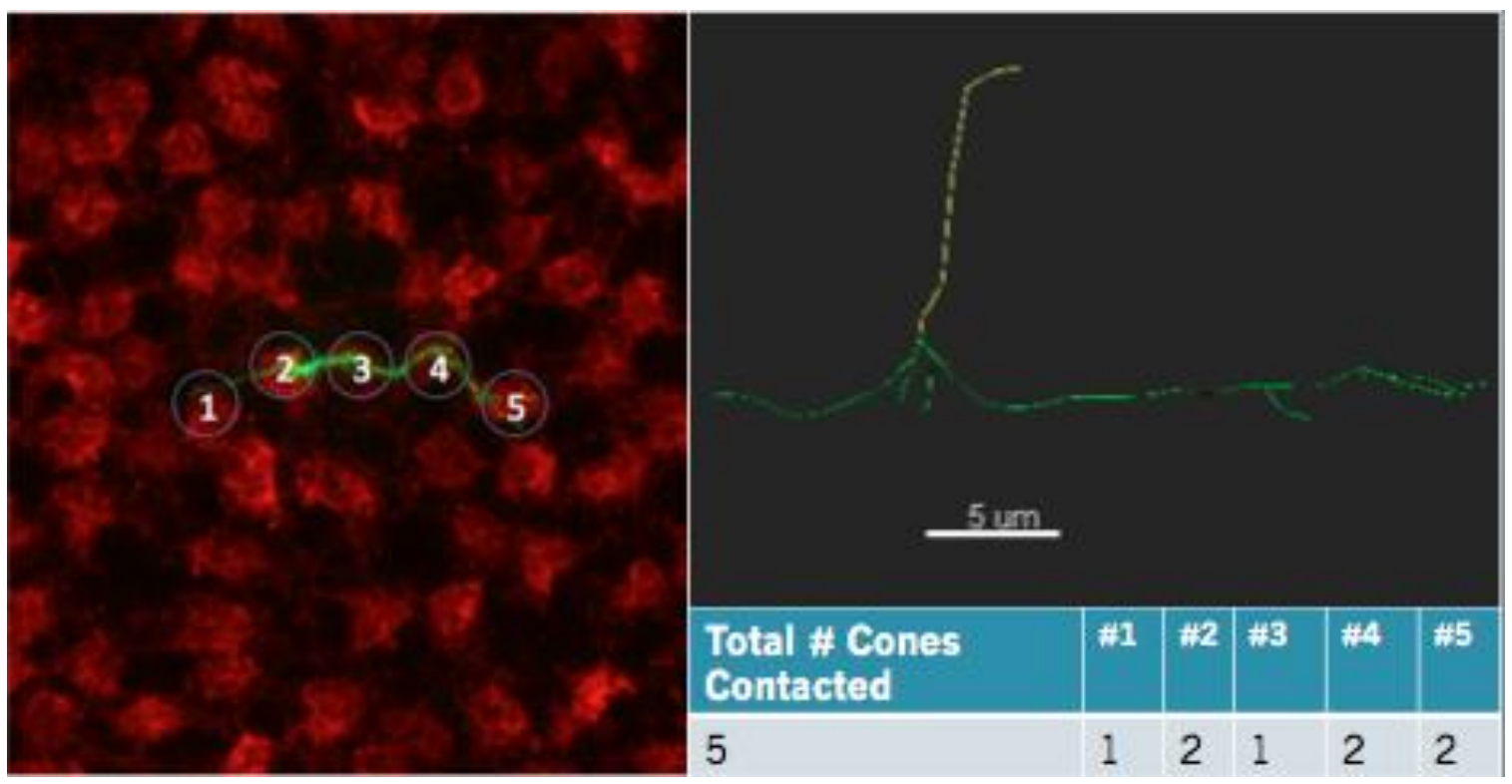

of three-dimensional images can be deceptive of cone contacts. Then the

number of dendritic contacts per cone terminal for each bipolar cell was recorded

(Figure 10).

Figure 10 Shows the manual cones per contact analysis, while showing the side view of the skeletonized bipolar cell dendrites to ensure that the dendrites were reaching down into the cone layer and were not premature sprouts off of the primary dendrite.

Cell data was cross referenced with similar cell data taken in a previous study months prior to make certain the measures were within reasonable realms. Data 
was also referenced against other paper's data to ensure the reliability of these measures.

Statistics. All statistical analyses were performed using Prism 5 (GraphPad Software, San Diego, CA) for WT VSX1 mice at P90 and P150, and the P150 GusGFP mice. The analysis of my study was modeled on a previous study done by Johnson and Kerschensteiner on retrograde plasticity of bipolar cells [13] as well as a study done by Wassle examining the bipolar cells of a retina, and in these studies an individual cell is considered a biological unit of analysis, not the animal or the retina that the cell came from. All distributions were tested for normality using the D'Agostino-Pearson omnibus normality test. Normally distributed data for the VSX1 WT at P90 and P150 and for the P150 GusGFP WT and P23H was analyzed using a two-tailed unpaired parametric ttest with Welch's Correction. Nonparametric statistical analyses for these two data sets were used when the distributions failed normality and a Mann-Whitney rank sum test was used. The P90 and P150 VSX1 mice data was compared to WT using the ANOVA test when data was normal. If data failed to be distributed normally, the data was compared using a nonparametric Kruskal-Wallis test, and Dunn's multiple comparison tests. Sholl analysis data was run through Two-way ANOVA tests. Any differences in data at $p \leq 0.05$ were considered significant.

\section{$\underline{\text { Results }}$}

Bipolar Cell Dendritic Morphology Differs at P90 and P150 VSX1 Mice 
Although I measured WT bipolar cells at P90 and P150 in VSX1 mice, all of my measures were similar (Figure 11), and I combined these data when comparing to the bipolar cells in P23H/VSX at P90 and P150.

The P90 P23H mice differ from the WT, as they show an increase in number of dendritic branch points Figure 12A). The P90 P23H VSX1 mice have no difference in the number of cones they contact when compared to the WT VSX1. They also do not differ from the wild type in the overall bipolar cell dendritic length, nor do they differ in number of contacts per cone.
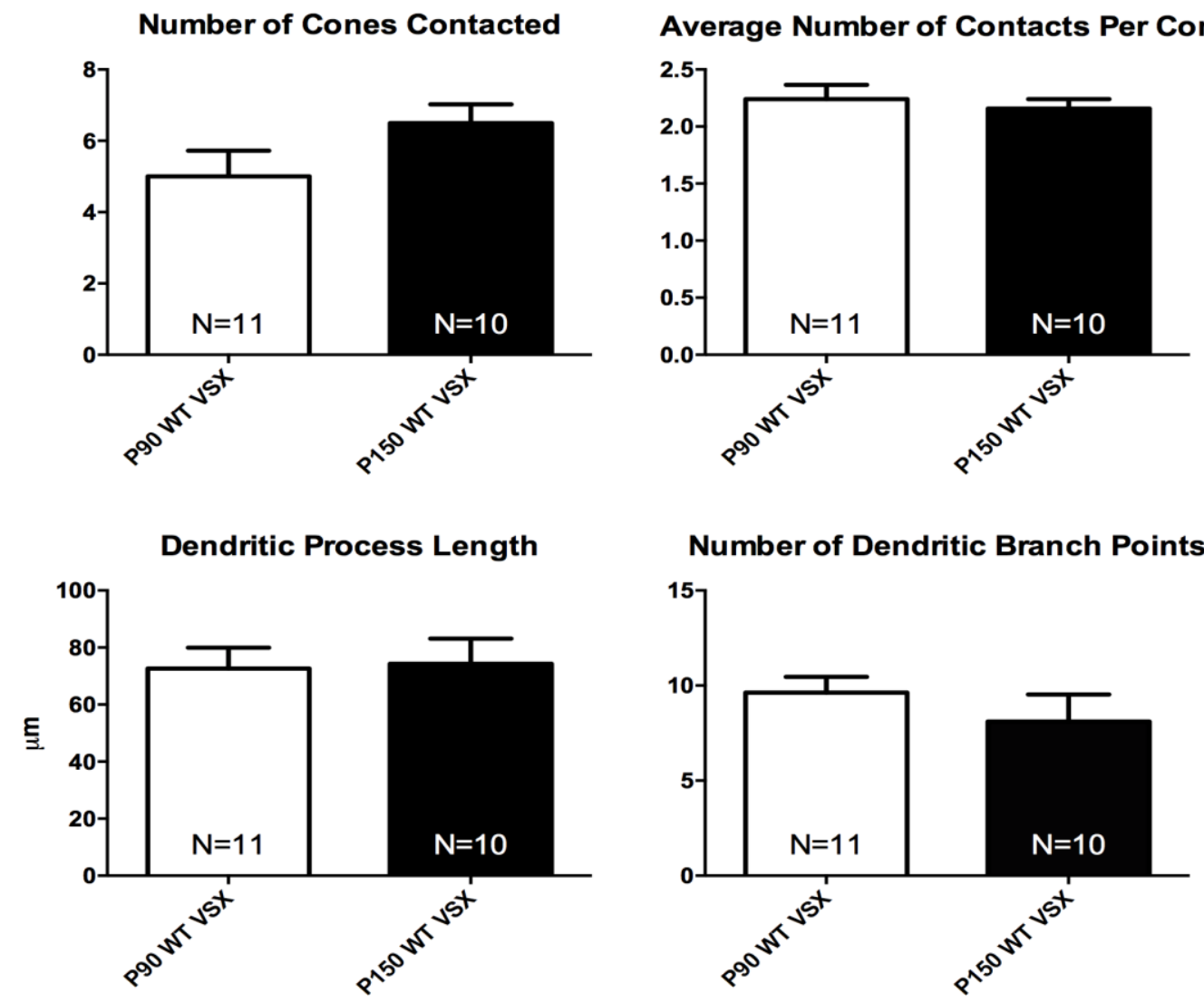

Figure 11 Shows that WT VSX1 bipolar cell morphology is similar at P90 and P150. As a consequence, I collapsed the WT data for comparison to the P23KI VSX1 data. 
The $\mathrm{P} 23 \mathrm{H}$ mice aged $\mathrm{P} 150$ show many morphological indications that

Number of Dendritic Branch Points
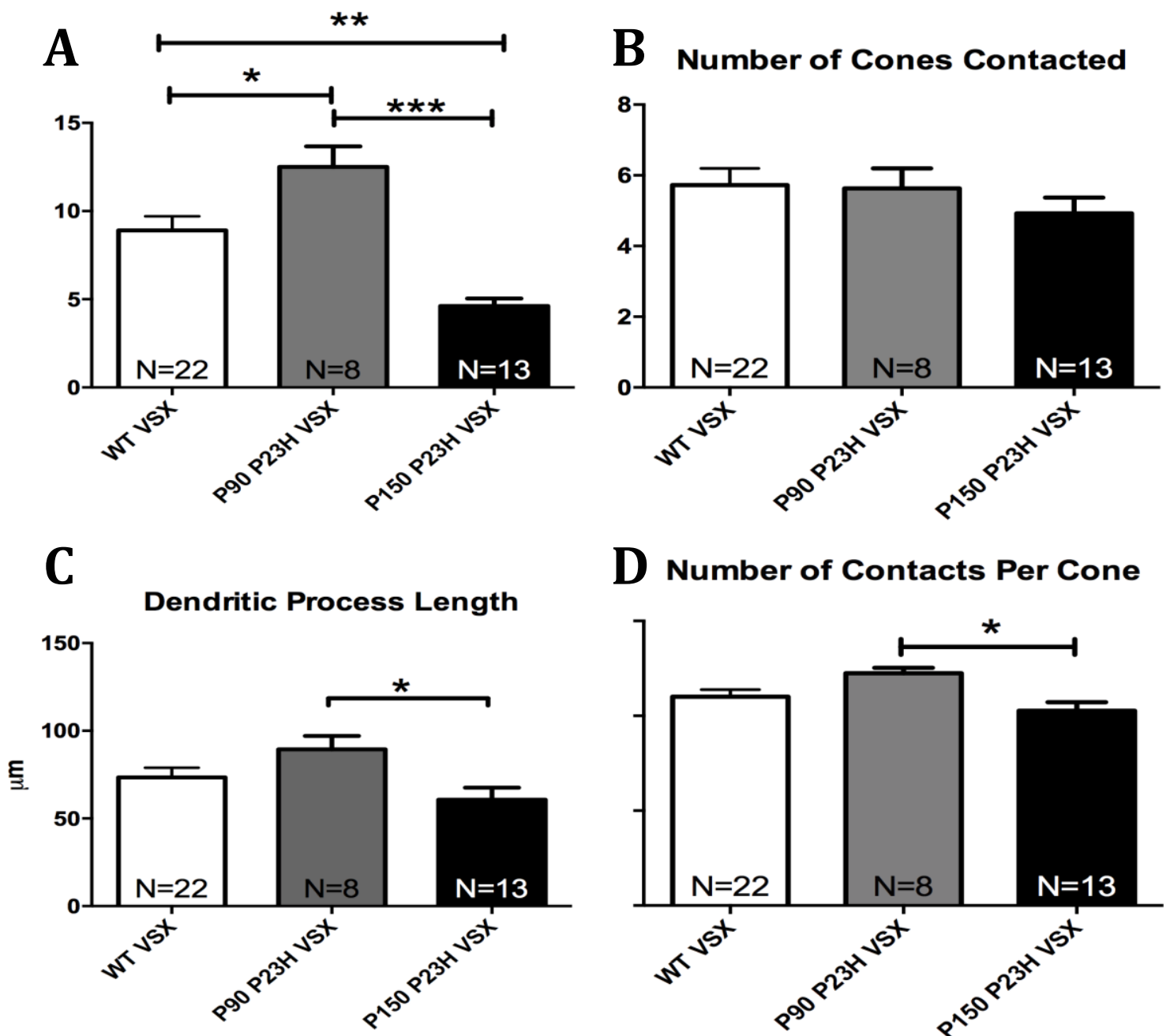

D Number of Contacts Per Cone

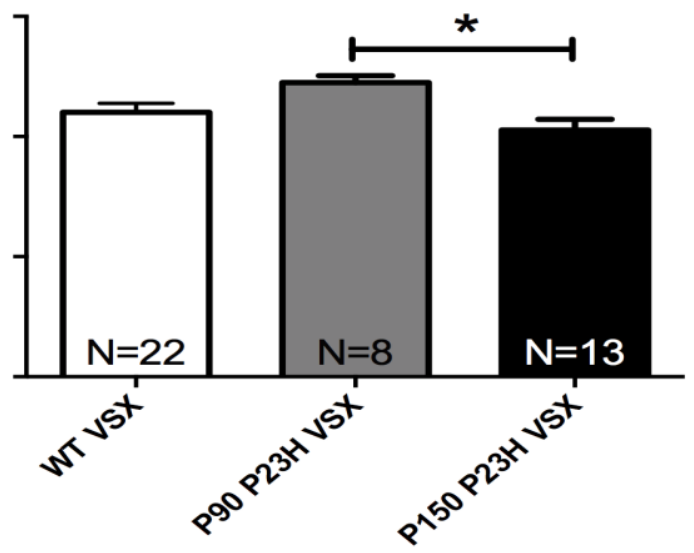

Figure 12 shows the morphological analysis data when comparing the VSX1 mice at the different time points from the wild types.

their dendrites are retracting compared to WT and to P90 bipolar cells. The P150 P23H mice differ from the WT in that they show a decrease in the number of dendritic branch points (Figure 12A). They do not differ in number of cones they 
contact compared to the WT VSX1, and P150 P23H VSX1 mice do not differ from the wild type in the overall bipolar cell dendritic length. Additionally, they do not differ from the WT in number of contacts per cone

Lastly, the P90 P23H mice differ from the P150 P23H mice in that the P150 mice have less branch points than the P90 P23H (One-Way ANOVA, $p<0.0001$ ), but they have no difference in the average number of cones the bipolar cells contact (Figure 12A and 12B). The P90 P23H VSX1 and P150 P23H VSX1 mice significantly differ from each other in dendritic process length, the mutated mice at P150 has an overall dendritic process length of approximately $15 \mu \mathrm{m}$ less than the P90 VSX1 mutated mice (Figure 12C). The P90 P23H differ from the P150 P23H mice in the number of contacts per cone. The mutated P150 mice tended to have about 0.4 less bipolar cell dendritic contacts per cone on average.

\section{GusGFP Bipolar Cells Number of Cones Contacted}

Bipolar Cell Dendritic Morphology

Between P150 WT and P23H

GusGFP Mice

The GusGFP mice at P150 show a nearly significant difference between the average numbers of cones contacted per bipolar cell. The P150 P23H GusGFP mice's bipolar cells tend to contact less

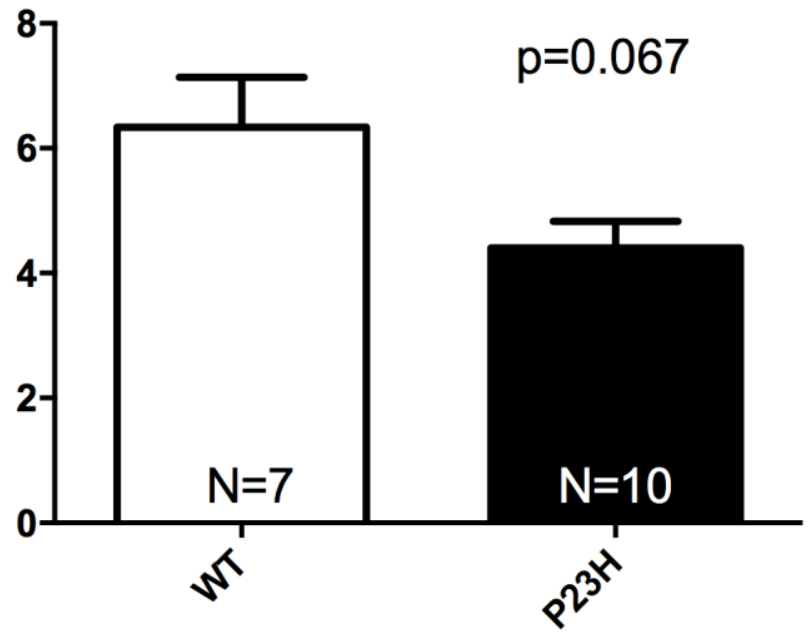

Figure 13 shows the data for the average number of Cones contacted per bipolar cell in the P150 GusGFP mice. 
cone terminals on average, $4.400 \pm 1.350$, than the P150 WT GusGFP mice's bipolar cells, $6.333 \pm 1.966$ (unpaired t-test, $P=0.07$ ). While I do not know, we think that statistical significance might be reached with a larger number of WT Gus-GFP bipolar cells, similar to the number that I had for the VSX1 bipolar cells.

It is important to note that with a larger sample there is a likelihood that the data could change and show different trends because all the data is very close to the line of significance.

\section{Discussion}

After looking at the WT, P23H P90, and P23H P150 VSX1 mice data, there seems to be multiple trends. The number of dendritic branch points increases compared to WT at P90 but the dendritic length does not change nor are more cones contacted. At P150, the dendritic process length, the number of branch points and the number of cones contacted all decrease compared to P90. What this seems to suggest is that the VSX bipolar cell's dendrites become more complex in the $\mathrm{P} 23 \mathrm{H} \mathrm{KI}$ retina at $\mathrm{P} 90$, which may mean that the cells put out more processes at this early stage of photoreceptor degeneration but do not seem to find cone contacts. The overall change in dendritic process VSX bipolar cells at P90 happens at a local level since the dendritic length does not change but the branch points are changing. The dendrites are rearranging and or changing in very localized ways versus branching out even further into the photoreceptor layer or attempting to contact more individual cone terminals. The number of cones contacted per bipolar cell does not change between the 
different groups of mice, however the number of individual bipolar cell contacts per cone does. The ANOVA test shows an overall difference, yet again there is no difference between P90 P23H and WT mice or P150 P23H and WT mice. The trend seen indicates that the P90 mutated mouse model increases its dendritic spread and cone contacts, from this is possible to infer that the bipolar cells are still in search of more or an optimal number of cone contacts. As degeneration proceeds, (P150), the VSX bipolar cell dendrites become less complex, retract and now lose contacts with cones. The significant drop in the dendritic branch points, length, and number of contacts per cone between the P90 and P150 suggests that the cell may have been unable to successfully maintain those local contacts that it extended, perhaps because it did not find a presynaptic cone. In other words, we can see that the main dendritic branches are not necessarily being affected by the retinal deterioration and that is proven by the maintenance of overall dendritic length and number of cones contacted, hence the localized dendritic changes in the cells.

Lastly, looking at the GusGFP data allows us to look at the ON pathway of cells. The bipolar cells showed no morphologically significant changes in the analyses, however, the GusGFP bipolar cells at P150 showed near significance in a difference between the numbers of cones contacted. The fact that the data is trending towards less bipolar cell contact with cones in the ON pathway suggests that the different pathways may go about retinal deterioration in different ways.

Retinitis pigmentosa (RP) is caused by different mutations in the retina that leads to photoreceptor death [9]. The bipolar cells that connect the rods and 
cones to the ganglion cells serve as the direct and primary connection for information flow from the photoreceptors to the rest of the brain [13]. The measured morphological changes in bipolar cells that signify an overall decrease of contact with the cone photoreceptors should play a part in the biological significance of the decrease of photopic vision in eyes with retinitis pigmentosa. The decrease in bipolar cell dendritic area and cone contacts over time should lead to a decrease in this information flow and therefore impaired vision. 


\section{$\underline{\text { References }}$}

1. Ronald G. Gregg, M.A.M., Stephen C. Massey, Function and Anatomy of the Mammalian Retina, in Retina, S.J. Ryan, Editor. 2013, Elsevier Saunders. p. 360-400.

2. Wässle, H., et al., Cone Contacts, Mosaics, and Territories of Bipolar Cells in the Mouse Retina. The Journal of Neuroscience, 2009. 29(1): p. 106-117.

3. Dryja, T.P., et al., A point mutation of the rhodopsin gene in one form of retinitis pigmentosa. Nature, 1990. 343(6256): p. 364-366.

4. Fransen, J.W., et al., Functional changes in $\mathrm{Tg}$ P23H-1 rat retinal responses: differences between $O N$ and OFF pathway transmission to the superior colliculus. Journal of Neurophysiology, 2015. 114(4): p. 2368-2375.

5. Ritter, E., M. Elgeti, and F.J. Bartl, Activity Switches of Rhodopsin. Photochemistry \& Photobiology, 2008. 84(4): p. 911-920.

6. Price, B.A., et al., Mislocalization and Degradation of Human P23H-RhodopsinGFP in a Knockin Mouse Model of Retinitis Pigmentosa. Investigative Ophthalmology \& Visual Science, 2011. 52(13): p. 9728-9736.

7. Ploss, A., Mouse models for human infectious diseases. Journal of Immunological Methods, 2014. 410: p. 1-2.

8. Hartong, D.T., E.L. Berson, and T.P. Dryja, Retinitis pigmentosa. The Lancet. 368(9549): p. 1795-1809.

9. Olsson, J.E.J., Transgenic mice with a rhodopsin mutation (Pro23His): a mouse model of autosomal dominant retinitis pigmentosa. Neuron. 9(5): p. 815-30.

10. Hoon, M., et al., Neurotransmission plays contrasting roles in the maturation of inhibitory synapses on axons and dendrites of retinal bipolar cells. Proceedings of the National Academy of Sciences, 2015. 112(41): p. 12840-12845.

11. Kolb, H., Cone Retinas Through the Retina. Webvision. The Organization of the Retina and Visual System, 2011.

12. Miyazaki, M., et al., Simia lentiviral vector-mediated retinal gene transfer of pigment epithelium-derived factor protects retinal degeneration and electrical defect in Royal College of Surgeons rats, Gene Therapy, 2003. 10: p.15031511.

13. Chen M, Wang K, Lin B (2012) Development and Degeneration of Cone Bipolar Cells Are Independent of Cone Photoreceptors in a Mouse Model of Retinitis Pigmentosa. PLoS ONE 7(8): e44036. doi:10.1371/journal.pone.0044036

14. Johnson, R., \& Kerschensteiner, D. (2014). Retrograde Plasticity and Differential Competition of Bipolar Cell Dendrites and Axons in the Developing Retina. Current Biology, 24(19), 2301-2306. doi:10.1016/j.cub.2014.08.018 